\title{
Prognostic Significance of COVID-19 Receptor ACE2 and Recommendation for Antihypertensive Drug in Renal Cell Carcinoma
}

\author{
Kihun Kim, ${ }^{1}$ Yeji Ko, ${ }^{2}$ Dai Sik Ko $\mathbb{D}^{3},{ }^{3}$ and Yun Hak Kim $\mathbb{D}^{4,5}$ \\ ${ }^{1}$ Department of Occupational and Environmental Medicine, Kosin University Gospel Hospital, Republic of Korea \\ ${ }^{2}$ Department of Statistics, University of Michigan, Michigan, USA \\ ${ }^{3}$ Division of Vascular Surgery, Department of Surgery, Gachon University Gil Medical Center, Republic of Korea \\ ${ }^{4}$ Department of Biomedical Informatics, School of Medicine, Pusan National University, Republic of Korea \\ ${ }^{5}$ Department of Anatomy, School of Medicine, Pusan National University, Republic of Korea
}

Correspondence should be addressed to Dai Sik Ko; daisik.ko@gilhospital.com and Yun Hak Kim; yunhak10510@pusan.ac.kr

Received 11 August 2020; Revised 9 November 2020; Accepted 17 November 2020; Published 27 November 2020

Academic Editor: Wen Shi

Copyright (c) 2020 Kihun Kim et al. This is an open access article distributed under the Creative Commons Attribution License, which permits unrestricted use, distribution, and reproduction in any medium, provided the original work is properly cited.

Purpose. Owing to its worldwide spread, the coronavirus disease (COVID-19) epidemic was declared a pandemic by the World Health Organization on March 11, 2020. Angiotensin-converting enzyme 2 (ACE2) is the outer surface protein of the cell membrane that is abundantly distributed in the heart, lungs, and kidneys and plays an important role in molecular docking of the severe acute respiratory syndrome coronavirus 2 . In this study, we aimed to analyze the difference in the survival rate according to ACE2 expressions in pan-cancer. Materials and Methods. We downloaded clinical and genomic data from The Cancer Genome Atlas. We used Kaplan-Meier with a log-rank test, and the Cox proportional hazards regression to analyze prognostic significance. Results. In the Kaplan-Meier curve, clear cell renal cell carcinoma (ccRCC), uveal melanoma, and prostate adenocarcinoma showed statistical significance. In the Cox regression, thyroid carcinoma and glioblastoma multiforme and ccRCC showed significant results. Only ccRCC had statistical significance, and high ACE2 expression is related to good prognosis. It is known that the ACE inhibitor, a primary antihypertensive agent, increases ACE2 expression. Conclusion. Based on these results, we believe that the ACE inhibitor will be important to increase the lifespan of ccRCC patients. This study is the first research to offer a recommendation on the use of anti-hypertensive drugs to ccRCC patients.

\section{Introduction}

Coronavirus (CoV) belongs to a family of viruses characterized by highly diverse, enveloped, positive-sense, and single-stranded RNA genomes [1]. They cause respiratory, gastrointestinal, hepatic, and neurological symptoms in animals or humans according to their type [2]. The wellknown Middle East respiratory syndrome $\mathrm{CoV}$ and severe acute respiratory syndrome $\mathrm{CoV}$ (SARS-CoV) are fatal to humans, while human $\mathrm{CoV}(\mathrm{HCoV}) \mathrm{OC} 43, \mathrm{HCoV}-229 \mathrm{E}$, and HCoV-NL63 cause only mild respiratory symptoms [1, 3-5]. In December 2019, cases of unknown pneumonia occurred in Wuhan, Hubei Province, China. Subsequently, the causative virus was extracted from human patients, and a molecular analysis revealed that it was a novel coronavirus [6]. The virus was tentatively named "severe acute respiratory syndrome coronavirus 2 (SARS-CoV-2)" by the International Committee on Taxonomy of Viruses [7]. The World Health Organization (WHO) named the disease caused by SARS-CoV-2 as COVID-19 on February 11, 2020 [8]. With its worldwide spread, the COVID-19 epidemic was declared a pandemic by the WHO on March 11, 2020 [9].

Angiotensin-converting enzyme 2 (ACE2) is the outer surface protein of the cell membrane that is abundantly distributed in the heart, lungs, and kidneys [10-12]. ACE2 is a functional receptor of SARS-CoV $[13,14]$. SARS-CoV-2 shares $80 \%$ similarity with the genome of SARS-CoV, and its cell entry mechanism is mediated by the ACE2 receptor 
$[13,15]$. ACE2 is currently emerging as a new research topic in the wake of SARS-CoV infection. The ACE inhibitor, which is widely used as a therapeutic agent for hypertension, is reported to upregulate the ACE2 receptor expression [16]. The use of ACE inhibitors has been suggested to increase the susceptibility to COVID-19 and worsen the COVID-19 outcome through an increase in the viral load [17]. However, owing to the insufficient evidence at present, the management of hypertension in patients with COVID-19 is controversial [18].

TCGA is a large prospective cohort with data on several variables (demographic, clinical, and genomic data) of approximately 11,000 patients for 33 common cancers [19]. Especially in the field of big data, high-dimensional genomics is available [20]. As the importance of managing cancer diseases increases in the COVID-19 pandemic era, we analyzed the difference in the survival rate according to ACE2 expression levels in 31 cancers by using The Cancer Genome Atlas (TCGA) dataset. Accordingly, we aimed to provide recommendations for the treatment of viral infection or use of ACE inhibitors in certain patients with cancer.

\section{Material and Methods}

2.1. Patients. The clinical and genomic data of 33 cancers listed in TCGA were downloaded from the Firehose database (https:/gdac.broadinstitute.org/) in February 2020. All TCGA data were available without restrictions from publications or presentations in accordance with TCGA publication guidelines. Patients' clinical variables such as cancer stage, age, sex, and censoring status, as well as ACE2 expression levels, were also extracted. Patient data with insufficient clinical or genetic information were excluded. Two cancers without ACE2 expression levels were excluded from the analysis.

2.2. Statistical Analyses. A violin plot with $\log 2$ transformed ACE2 expression on the $y$-axis and cancer types on the $x$ -axis was created to compare the gene expression levels between the different cancers (Figure 1). In the present study, we performed a Kaplan-Meier analysis and log-rank tests. A continuous ACE2 expression level was converted to a binary factor low (0) and high (1), with a median cutoff; two distinct survival distributions expressed in binary form were developed. Cancers with positive and negative relationships with the ACE2 expression level and survival rate were defined as "positive" and "negative" cancers, respectively. Nineteen cancers were positive, and 12 were negative on the basis of the odds ratios.

We also used univariable and multivariable Cox proportional hazards regression models for all 31 cancers to estimate the hazard ratio, 95\% confidence interval, and $p$ value of ACE2 and the other clinical variables in the data for each cancer as we described previously [20, 21]. Finally, we checked the significance of the values and combined the results with the survival analysis output to conclude which type of cancer could be most affected by the ACE2 gene. The concordance index was used to evaluate the prediction accuracy of our statistical models. A flowchart is provided in Figure 2 to visually represent the process. All statistical analyses were performed using $\mathrm{R}$ version 3.6.0 ( $\mathrm{R}$ Foundation for Statistical Computing, Vienna, Austria).

\section{Results}

3.1. Patient Data and ACE2 Expression. The numbers of samples obtained from TCGA dataset for all cancer types are shown in Table 1. Demographic and clinical details are not shown in this paper. Two cancers, clear cell renal cell carcinoma and renal papillary cell carcinoma, stood out in the violin plot (Figure 1) because their median log2 transformed ACE2 expression levels were $>10$. Eight cancers, namely, brain lower-grade glioma, invasive breast carcinoma, uveal melanoma, glioblastoma multiforme, mesothelioma, pheochromocytoma and paraganglioma, sarcoma, and skin melanoma, were found to have median values of $<2.5$.

3.2. Survival Analyses. None of the negative cancers had significant log-rank test results. Of the 19 positive cancers, three, namely clear cell renal cell carcinoma, uveal melanoma, and prostate adenocarcinoma, had $p$ values of $<0.05$ and thus were considered significant in the Kaplan-Meier analysis and log-rank test (Figure 2). The survival plots of these cancers are shown in Figure 3.

Although two negative cancers, thyroid carcinoma and glioblastoma multiforme, and two positive cancers, adrenocortical carcinoma and mesothelioma, were significant in the multivariable regression results, they were still insignificant in the univariable Cox regression models (Figure 2). As the multivariable models of these cancers could elicit a false idea of interaction effects between covariates, we decided not to further investigate them.

Clear cell renal cell carcinoma was the only cancer with significant results in both the univariable and multivariable Cox regression analyses (Figure 2). The cancer had hazard ratios of 0.571884 and 0.61273 in the univariable and multivariable Cox regression models, respectively. These values indicate that the relative risks of death in the defined period were approximately $43 \%$ and $39 \%$ lower, respectively, in the high gene expression group than in the low gene expression group. These results were also observed in a forest plot, as only clear cell renal cell carcinoma had a confidence interval of $<0$ in both the univariable and multivariable regression analyses (Figures 4 and 5).

The concordance indexes of the univariable Cox regression models for clear cell renal cell carcinoma, uveal melanoma, and prostate adenocarcinoma were $0.6874,0.59$, and 0.5351 , respectively (Table 2 ). As a model with a concordance index of $>0.55$ is considered a good model, this implies a good predictive ability for clear cell renal cell carcinoma and uveal melanoma [22]. We conclude that high ACE2 gene expression levels could positively influence the survival rate of patients with clear cell renal cell carcinoma and have high model accuracy levels.

\section{Discussion}

In this study, we analyzed the survival rate according to ACE2 expression level for various cancers by using statistical 


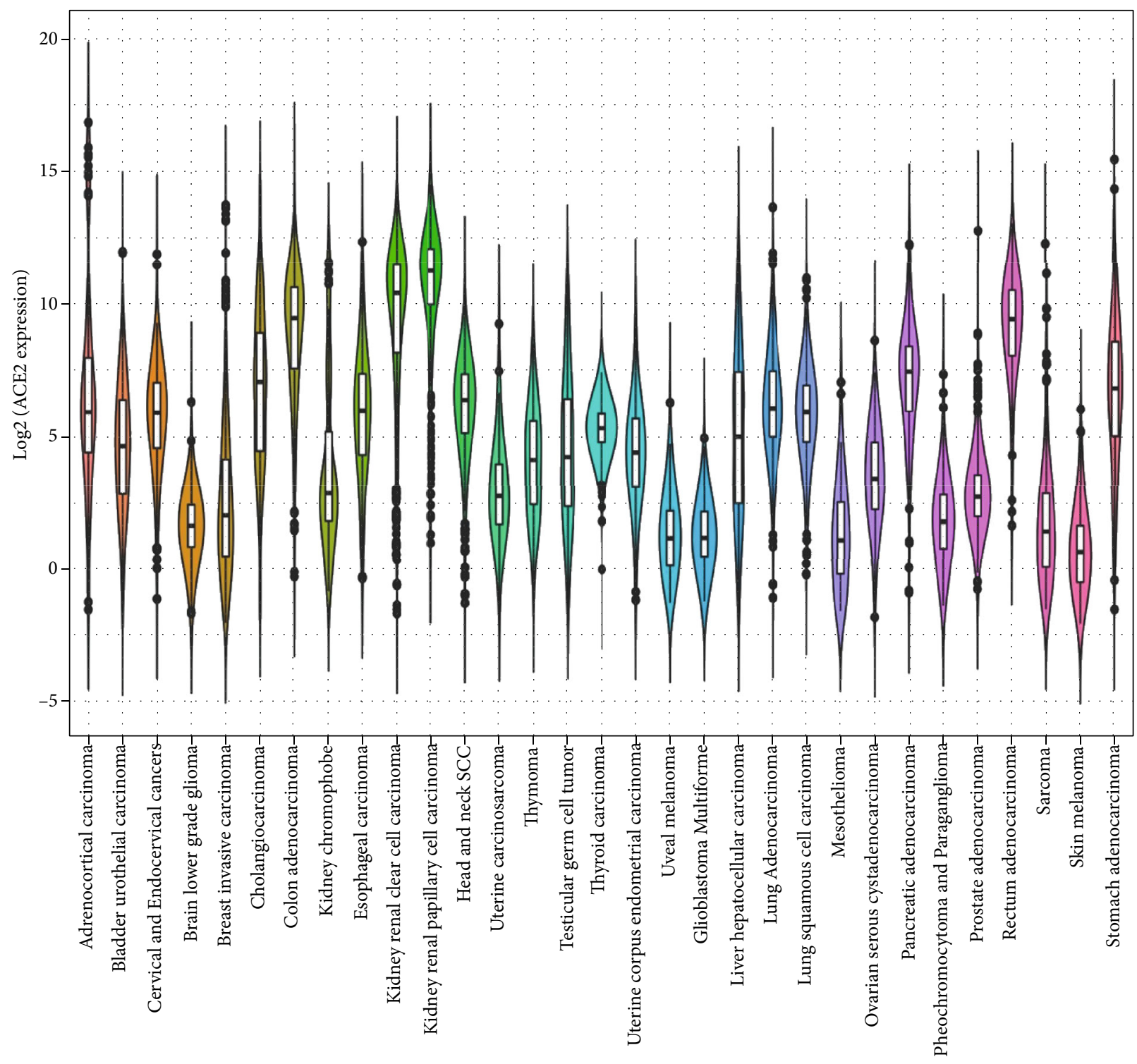

Cancer type

Cancer type

$\square$ Adrenocortical carcinoma

Bladder urothelial carcinoma

$\square$ Cervical and Endocervical cancers

Brain lower grade glioma

Breast invasive carcinoma

Cholangiocarcinoma

$\square$ Colon adenocarcinoma

Kidney chromophobe

Esophageal carcinoma

Kidney renal clear cell carcinoma

Kidney renal papillary cell carcinoma
Head and neck SCC

Uterine carcinosarcoma

Thymoma

Testicular germ cell tumor

Thyroid carcinoma

Uterine corpus endometrial carcinoma

Uveal melanoma

Glioblastoma Multiforme

Liver hepatocellular carcinoma

$\square$ Lung Adenocarcinoma

Lung squamous cell carcinoma
Mesothelioma

$\square$ Ovarian serous cystadenocarcinoma

Pancreatic adenocarcinoma

Pheochromocytoma and Paraganglioma

Prostate adenocarcinoma

Rectum adenocarcinoma

$\square$ Sarcoma

$\square$ Skin melanoma

$\square$ Stomach adenocarcinoma

Figure 1: Violin plot of the ACE2 expression levels ( $\log 2$ transformation). 


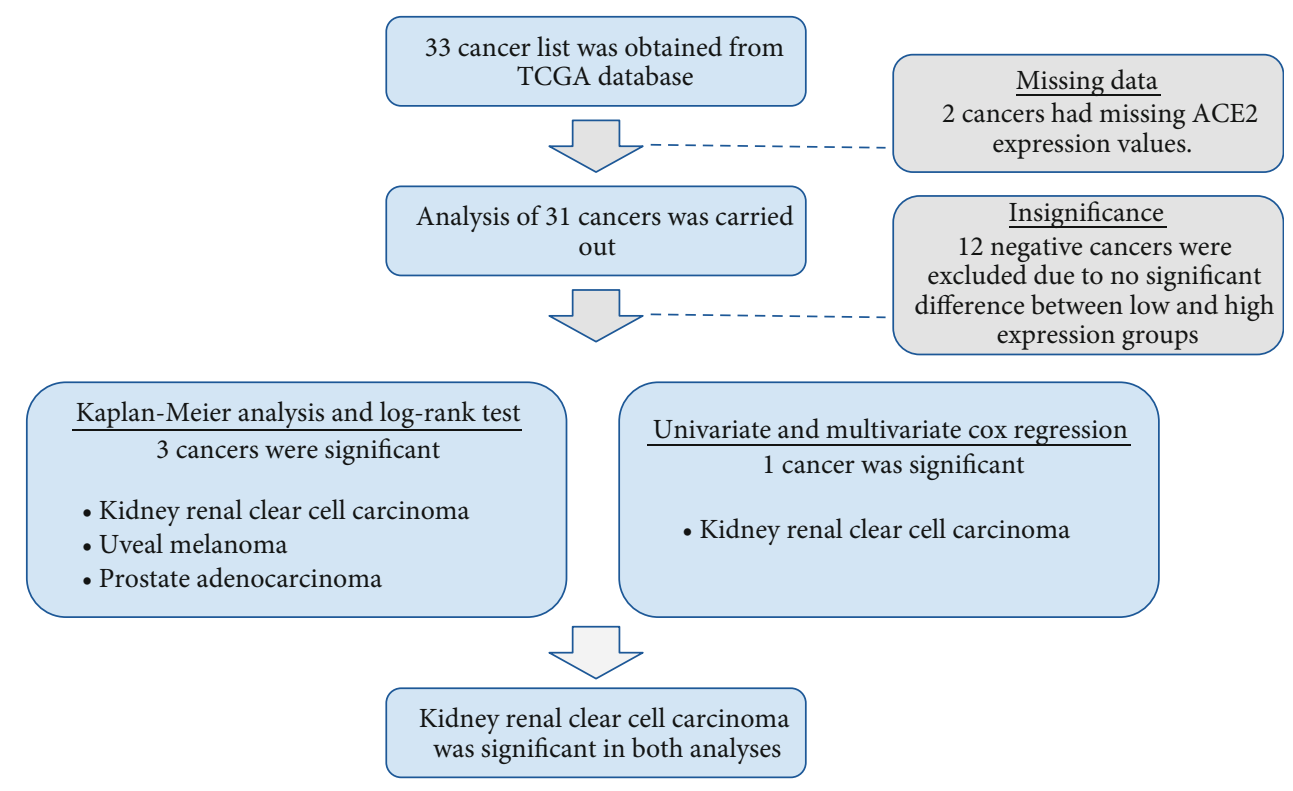

Figure 2: Flowchart for statistical analysis.

TABle 1: Number of samples.

\begin{tabular}{|c|c|c|c|}
\hline Cancer type & Number $(n)$ & Cancer type & Number $(n)$ \\
\hline Adrenocortical carcinoma & 68 & Uterine corpus endometrial carcinoma & 144 \\
\hline Bladder urothelial carcinoma & 425 & Uveal melanoma & 74 \\
\hline Cervical and endocervical cancers & 276 & Glioblastoma multiforme & 143 \\
\hline Brain lower-grade glioma & 418 & Liver hepatocellular carcinoma & 359 \\
\hline Breast invasive carcinoma & 985 & Lung adenocarcinoma & 367 \\
\hline Cholangiocarcinoma & 36 & Lung squamous cell carcinoma & 539 \\
\hline Colon adenocarcinoma & 313 & Mesothelioma & 86 \\
\hline Kidney chromophobe & 89 & Ovarian serous cystadenocarcinoma & 277 \\
\hline Esophageal carcinoma & 172 & Pancreatic adenocarcinoma & 163 \\
\hline Clear cell renal cell carcinoma & 583 & Pheochromocytoma and paraganglioma & 187 \\
\hline Renal papillary cell carcinoma & 282 & Prostate adenocarcinoma & 494 \\
\hline Head and neck squamous cell carcinoma & 463 & Rectal adenocarcinoma & 82 \\
\hline Uterine carcinosarcoma & 46 & Sarcoma & 253 \\
\hline Thymoma & 117 & Skin melanoma & 421 \\
\hline Testicular germ cell tumor & 133 & Stomach adenocarcinoma & 329 \\
\hline Thyroid carcinoma & 488 & & \\
\hline
\end{tabular}

models such as the Kaplan-Meier analysis and log-rank test and univariable and multivariable Cox regression analyses. In addition, the survival prediction performance of ACE2 expression level was evaluated with the concordance index to corroborate the significantly different results from the previous tests.

The results of the Kaplan-Meier analysis and log-rank test indicated significant differences in survival rates among patients with renal cell carcinoma, uveal melanoma, and prostate adenocarcinoma according to ACE2 expression level. The results of the univariable and multivariable Cox regression analyses indicated that the ACE2 expression level had a significant effect on the survival rates of patients with renal clear cell carcinoma, thyroid carcinoma, and glioblastoma multiforme. Only clear cell renal cell carcinoma showed significant results in both the Kaplan-Meier analysis and log-rank test/univariable and multivariable Cox regression analyses. Therefore, we could assume that in clear cell renal cell carcinoma, the ACE2 expression level is strongly associated with survival rate and acts as an important causal factor for predicting the survival rate. The concordance index of clear cell renal cell carcinoma was 0.687 , confirming its good survival prediction performance. As the hazard ratio of clear cell renal cell carcinoma was significantly $<1$ in both the univariable and multivariable Cox regression analyses, we estimated 
Kaplan-Meier plot
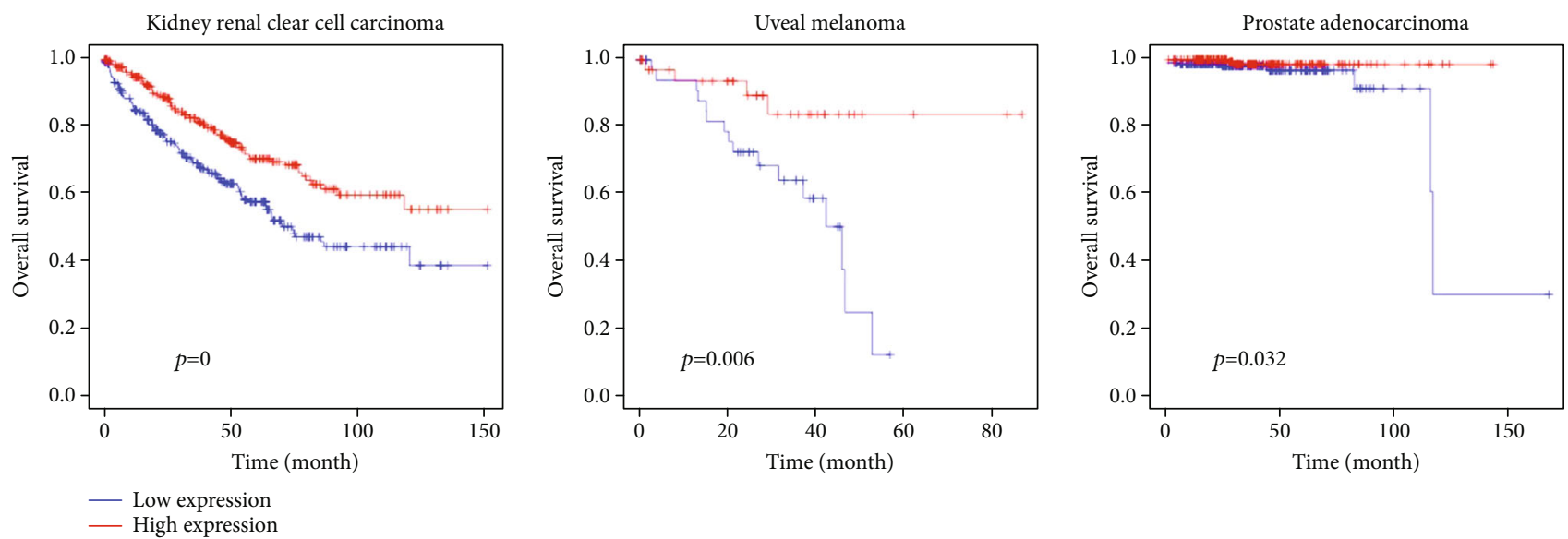

FIgURE 3: Survival plots of patients with cancers who had significant log-rank test results.

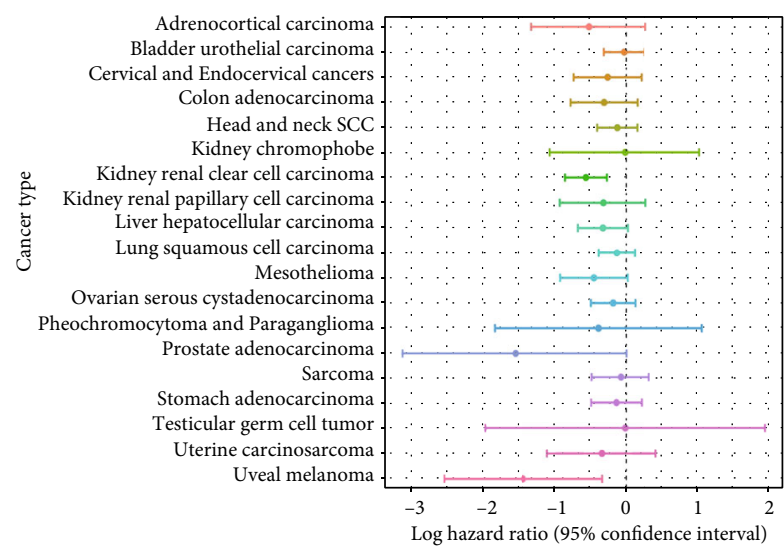

FIGURE 4: Forest plot of the hazard ratios (univariable) of 31 cancers.

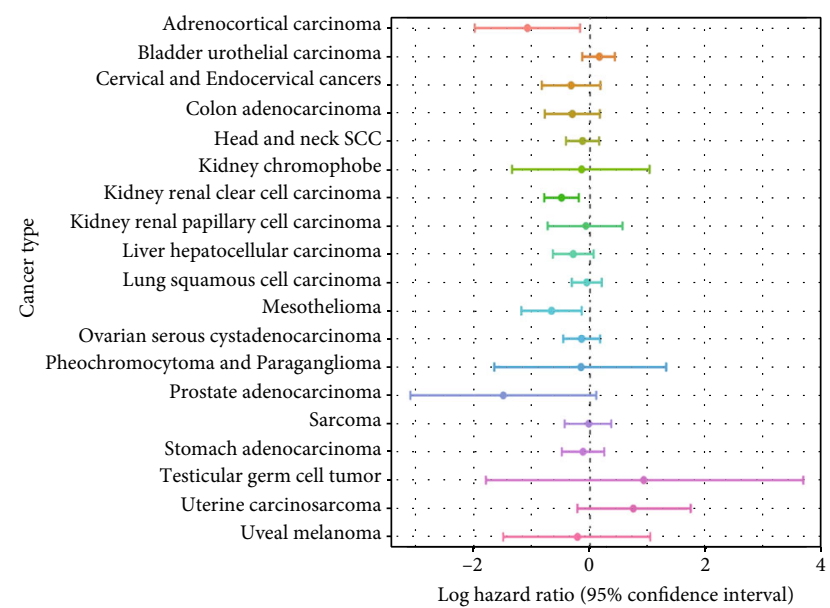

Figure 5: Forest plot of the hazard ratios (multivariable) of 31 cancers.

that the higher the patient's ACE2 expression level, the lower the patient's estimated risk, which corresponds to a higher survival rate.
TABLE 2: Concordance index scores.

\begin{tabular}{lc}
\hline Cancer type & Concordance index score \\
\hline Renal cell clear cell carcinoma & 0.687 \\
Uveal melanoma & 0.590 \\
Prostate adenocarcinoma & 0.535
\end{tabular}

The ACE inhibitor is used as an initial therapy for high blood pressure in many situations (e.g., heart failure with reduced ejection fraction and chronic kidney disease) [2326]. It inhibits the conversion of angiotensin I to angiotensin II to reduce the activity of the renin-angiotensin-aldosterone system [27, 28]. The use of ACE inhibitor has been shown to increase the ACE2 expression level in human and animal studies $[16,29]$. Therefore, we assumed that the use of ACE inhibitors for controlling high blood pressure in patients with clear cell renal cell carcinoma will increase their ACE2 expression levels, which may help to improve survival rates if the administration of the ACE inhibitor is prioritized when choosing a high blood pressure control drug for these patients.

As mentioned earlier, ACE2, which is known to play an important role in molecular docking in the cell entry process of CoVs, is associated with mainly respiratory symptoms [2, 30]. In addition, an increased ACE2 expression level induces more CoV viral loads, including those of SARS-CoV and SARS-CoV-2. Cancers with significant results in this study were less relevant to respiratory viruses. Therefore, ACE2 expression level and survival rate seem to have no significant correlation in patients with respiratory cancer. The cancers with significant results in this study are presumed to be less relevant to respiratory viruses, including CoVs. Therefore, we speculated that SARS-CoV infection does not have a significant effect on the survival rate of certain cancer patients.

In several carcinomas (breast cancer, uterus corpus endometrial carcinoma, kidney renal papillary carcinoma, nonsmall-cell lung cancer, hepatocellular carcinoma, and pancreatic cancer), ACE2 expression was downregulated, which 


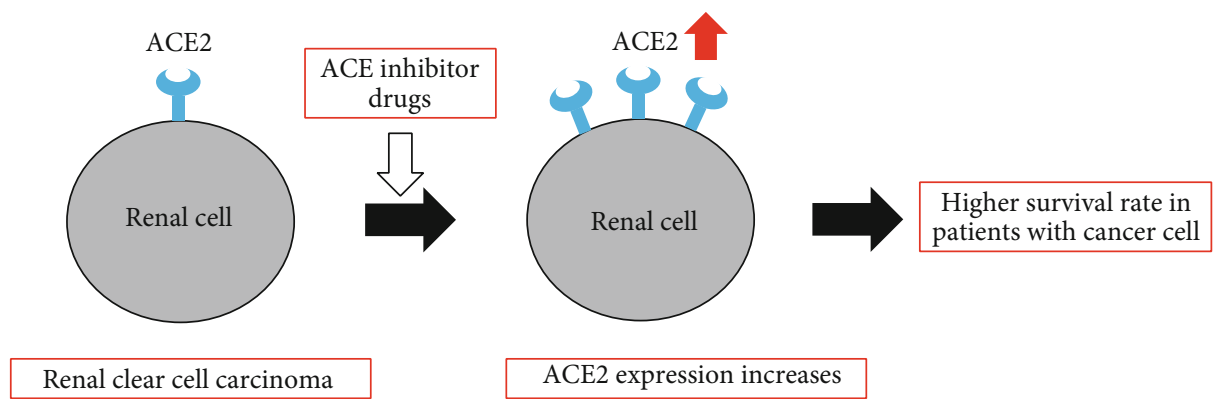

FIGURE 6: Graphical summary of this study.

is thought to be a poor prognostic factor [31-35]. This is consistent with the content of our study. In a recent paper, CD4 memory, CD8 effector, T helper cell, dendritic cell, and NK cell, which are associated with tumor infiltration, are reported to increase in ccRCC tissues [31]. The decrease in ACE2 expression is related to tumor proliferation, stemness, and epithelial-mesenchymal transition even at the micro environmental level [36]. It was assumed that the change in the immune microenvironment resulted in a difference in survival rates, and the increase in ACE2 expression is thought to mainly play an antitumor role. However, to the author's knowledge, it is assumed that the clear mechanisms of ACE2 expression and ccRCC are not established. The further clinical validation study that correlated between ACE2 expression and survival rates is required.

The limitation of this study is that the statistical analyses were conducted only with a single cohort. The results must be verified through a multicohort analysis. Second, the suggested drugs for hypertension and viral infections were hypothesized through statistical results, and the actual clinical outcomes require validation through further clinical trials. Third, because the number of samples collected for each cancer varied (range, 68-985), the power of the statistical results also varied among the cancer types.

\section{Conclusion}

By using big data, we analyzed the differences in survival rates among certain cancers according to ACE2 expression, which is known to be important for the entry of SARSCoV2, the virus responsible for the COVID-19 pandemic. The ACE2 expression level was highly relevant to the survival rate of patients with clear cell renal cell carcinoma and could be an important factor for predicting survival. The results of this study might be useful for further studying ACE-related treatments, care, and outcomes in patients with ccRCC (Figure 6).

\section{Data Availability}

Data are available upon TCGA publication guideline.

\section{Conflicts of Interest}

The authors declare that there is no conflict of interest regarding the publication of this paper.

\section{Authors' Contributions}

$\mathrm{KK}, \mathrm{YK}, \mathrm{DK}$, and YK designed this study. KK and YK collected and analyzed the data. KK, YK, and DK checked the statistical analysis. KK and YK wrote the manuscript. DK and YK revised the manuscript. Kihun Kim and Yeji Ko contributed equally to this work as first authors.

\section{Acknowledgments}

This work was supported by the Medical Research Center (MRC) program (NRF-2018R1A5A2023879), Basic Science Research Program (NRF-2020R1C1C1003741), and Collaborative Genome Program for Fostering New Post-Genome Industry (NRF-2017M3C9A6047610) through a National Research Foundation of Korea (NRF) grant funded by the Korean Government (MOE).

\section{References}

[1] A. Zumla, J. F. Chan, E. I. Azhar, D. S. Hui, and K.-Y. Yuen, "Coronaviruses - drug discovery and therapeutic options," Nature Reviews Drug Discovery, vol. 15, no. 5, pp. 327-347, 2016.

[2] F. He, Y. Deng, and W. Li, "Coronavirus disease 2019: what we know?," Journal of Medical Virology, vol. 92, no. 7, pp. 719$725,2020$.

[3] R. Channappanavar, J. Zhao, and S. Perlman, "T cell-mediated immune response to respiratory coronaviruses," Immunologic Research, vol. 59, no. 1-3, pp. 118-128, 2014.

[4] V. C. C. Cheng, S. K. P. Lau, P. C. Y. Woo, and K. Y. Yuen, "Severe acute respiratory syndrome coronavirus as an agent of emerging and reemerging infection," Clinical Microbiology Reviews, vol. 20, no. 4, pp. 660-694, 2007.

[5] J. F. W. Chan, S. K. P. Lau, K. K. W. To, V. C. C. Cheng, P. C. Y. Woo, and K. Y. Yuen, "Middle East respiratory syndrome coronavirus: another zoonotic betacoronavirus causing SARS-like disease," Clinical Microbiology Reviews, vol. 28, no. 2, pp. 465-522, 2015.

[6] J. Sun, W. T. He, L. Wang et al., "COVID-19: epidemiology, evolution, and cross-disciplinary perspectives," Trends in Molecular Medicine, vol. 26, no. 5, pp. 483-495, 2020.

[7] A. E. Gorbalenya, Severe Acute Respiratory Syndrome-Related Coronavirus-The Species and its Viruses, a Statement of the Coronavirus Study Group, BioRxiv, 2020.

[8] S. Zhao, Q. Lin, J. Ran et al., "Preliminary estimation of the basic reproduction number of novel coronavirus (2019-nCoV) 
in China, from 2019 to 2020: a data-driven analysis in the early phase of the outbreak," International Journal of Infectious Diseases, vol. 92, pp. 214-217, 2020.

[9] A. A. A. Anjorin, "The coronavirus disease 2019 (COVID-19) pandemic: a review and an update on cases in Africa," Asian Pacific Journal of Tropical Medicine, vol. 13, no. 5, p. 199, 2020.

[10] I. Hamming, W. Timens, M. Bulthuis, A. Lely, G. Navis, and H. van Goor, "Tissue distribution of ACE2 protein, the functional receptor for SARS coronavirus. A first step in understanding SARS pathogenesis," The Journal of Pathology, vol. 203, no. 2, pp. 631-637, 2004.

[11] M. Donoghue, F. Hsieh, E. Baronas et al., "A novel angiotensin-converting enzyme-related carboxypeptidase (ACE2) converts angiotensin I to angiotensin 1-9," Circulation Research, vol. 87, no. 5, pp. e1-e9, 2000.

[12] C. Baraniuk, Receptor for SARS-CoV-2 Present in Wide Variety of Human Cells, The Scientist Magazine, 2020.

[13] R. Yan, Y. Zhang, Y. Li, L. Xia, Y. Guo, and Q. Zhou, "Structural basis for the recognition of SARS-CoV-2 by full-length human ACE2," Science, vol. 367, no. 6485, pp. 1444-1448, 2020.

[14] J. Guo, X. Wei, Q. Li et al., "Single-cell RNA analysis on ACE2 expression provides insights into SARS-CoV-2 potential entry into the bloodstream and heart injury," Journal of Cellular Physiology, vol. 235, no. 12, pp. 9884-9894, 2020.

[15] W. Li, M. J. Moore, N. Vasilieva et al., “Angiotensin-converting enzyme 2 is a functional receptor for the SARS coronavirus," Nature, vol. 426, no. 6965, pp. 450-454, 2003.

[16] C. M. Ferrario, J. Jessup, M. C. Chappell et al., "Effect of angiotensin-converting enzyme inhibition and angiotensin II receptor blockers on cardiac angiotensin-converting enzyme 2," Circulation, vol. 111, no. 20, pp. 2605-2610, 2005.

[17] G. M. Kuster, O. Pfister, T. Burkard et al., "SARS-CoV2: should inhibitors of the renin-angiotensin system be withdrawn in patients with COVID-19?," European Heart Journal, vol. 41, no. 19, pp. 1801-1803, 2020.

[18] A. B. Patel and A. Verma, "COVID-19 and angiotensinconverting enzyme inhibitors and angiotensin receptor blockers: what is the evidence?," JAMA, vol. 323, no. 18, pp. 1769-1770, 2020.

[19] J. Liu, T. Lichtenberg, K. A. Hoadley et al., "An integrated TCGA pan-cancer clinical data resource to drive high-quality survival outcome analytics," Cell, vol. 173, no. 2, 2018.

[20] K. Pak, S. O. Oh, T. S. Goh et al., "A user-friendly, web-based integrative tool (ESurv) for survival analysis: development and validation study," Journal of Medical Internet Research, vol. 22, no. 5, article e16084, 2020.

[21] T. S. Goh, J. S. Lee, J. Il Kim et al., "Prognostic scoring system for osteosarcoma using network-regularized high-dimensional Cox-regression analysis and potential therapeutic targets," Journal of Cellular Physiology, vol. 234, no. 8, pp. 1385113857, 2018.

[22] F. E. Harrell, K. L. Lee, and D. B. Mark, "Multivariable prognostic models: issues in developing models, evaluating assumptions and adequacy, and measuring and reducing errors," Statistics in Medicine, vol. 15, no. 4, pp. 361-387, 1996.

[23] A. V. Chobanian, G. L. Bakris, H. R. Black et al., "The seventh report of the joint national committee on prevention, detection, evaluation, and treatment of high blood pressure: the JNC 7 report," JAMA, vol. 289, no. 19, pp. 2560-2572, 2003.

[24] G. Mancia, R. Fagard, K. Narkiewicz et al., "2013 practice guidelines for the management of arterial hypertension of the
European Society of Hypertension (ESH) and the European Society of Cardiology (ESC)," Journal of Hypertension, vol. 31, no. 10, pp. 1925-1938, 2013.

[25] C. Rosendorff, H. R. Black, C. P. Cannon et al., "Treatment of hypertension in the prevention and management of ischemic heart disease: a scientific statement from the American Heart Association Council for High Blood Pressure Research and the Councils on Clinical Cardiology and Epidemiology and Prevention," Circulation, vol. 115, no. 21, pp. 2761-2788, 2007.

[26] B. Williams, G. Mancia, W. Spiering et al., "2018 ESC/ESH guidelines for the management of arterial hypertension," European Heart Journal, vol. 39, no. 33, pp. 3021-3104, 2018.

[27] K. Jandeleit-Dahm and M. E. Cooper, "Hypertension and diabetes: role of the renin-angiotensin system," Endocrinology and Metabolism Clinics, vol. 35, no. 3, pp. 469-490, 2006.

[28] W. Wang, S. M. K. McKinnie, M. Farhan et al., "Angiotensinconverting enzyme 2 metabolizes and partially inactivates pyrapelin-13 and apelin-17: physiological effects in the cardiovascular system," Hypertension, vol. 68, no. 2, pp. 365-377, 2016.

[29] M. Furuhashi, N. Moniwa, T. Mita et al., "Urinary angiotensin-converting enzyme 2 in hypertensive patients may be increased by olmesartan, an angiotensin II receptor blocker," American Journal of Hypertension, vol. 28, no. 1, pp. 15-21, 2015.

[30] Y. Zhang, N. Zheng, P. Hao, Y. Cao, and Y. Zhong, "A molecular docking model of SARS-CoV S1 protein in complex with its receptor, human ACE2," Computational Biology and Chemistry, vol. 29, no. 3, pp. 254-257, 2005.

[31] W. Yang, L. Li, K. Zhang et al., "ACE2 correlated with immune infiltration serves as a novel prognostic biomarker in clear cell renal cell carcinoma: implication for COVID-19," International Journal of Biological Sciences, vol. 17, no. 1, pp. 20-31, 2021.

[32] Q. Zhang, S. Lu, T. Li et al., "ACE2 inhibits breast cancer angiogenesis via suppressing the VEGFa/VEGFR2/ERK pathway," Journal of Experimental \& Clinical Cancer Research, vol. 38, no. 1, p. 173, 2019.

[33] Y. Feng, H. Wan, J. Liu et al., "The angiotensin-converting enzyme 2 in tumor growth and tumor-associated angiogenesis in non-small cell lung cancer," Oncology Reports, vol. 23, no. 4, pp. 941-948, 2010.

[34] L. Zhou, R. Zhang, W. Yao et al., "Decreased expression of angiotensin-converting enzyme 2 in pancreatic ductal adenocarcinoma is associated with tumor progression," The Tohoku Journal of Experimental Medicine, vol. 217, no. 2, pp. 123-131, 2009.

[35] G. Ye, Y. Qin, X. Lu et al., "The association of reninangiotensin system genes with the progression of hepatocellular carcinoma," Biochemical and Biophysical Research Communications, vol. 459, no. 1, pp. 18-23, 2015.

[36] Z. Zhang, L. Li, M. Li, and X. Wang, “The SARS-CoV-2 host cell receptor ACE2 correlates positively with immunotherapy response and is a potential protective factor for cancer progression," Computational and Structural Biotechnology Journal, vol. 18, pp. 2438-2444, 2020. 